\title{
Lower segment caesarian section \& morbidly adherent placenta
}

\author{
P Thulasi ${ }^{1{ }^{1 *},}$ M Shanthi. $\mathbf{M}^{2}$ \\ ${ }^{1}$ Professor, ${ }^{2}$ Senior Resident, Dept. of Obstetrics and Gynaecology, P K DAS Institute of Medical Sciences, Kerala, \\ India
}

*Corresponding Author:

Email: drpthulasi@ rediffmail.com

\begin{abstract}
The incidence of placenta accreta has increased dramatically over the last three decades, in concert with increase in caesarean delivery rate. ${ }^{1}$ Placenta previa and caesarean section are significant risk factors. Morbidly adherent placenta is associated with high fetomaternal morbidity and mortality.
\end{abstract}

Keywords: Caesarian section, Placenta previa, Morbidly adherent placenta, Ultrasound.

\section{Case History}

A 27 year old lady, married for 5years, $\mathrm{G}_{4} \mathrm{P}_{1} \mathrm{~L}_{1} \mathrm{~A}_{1} \mathrm{E}_{1}$ case of previous LSCS who underwent MTP 10days back presented to our causality with acute lower abdominal pain, mid bleeding per vaginum and giddiness.

Her past obstetric history shows that she had an ectopic pregnancy first diagnosed at 7 wks of gestation for which she underwent laparoscopic Salpingectomy, next was spontaneous abortion at 6 wks. The last one was an Emergency LSCS for fetal distress with live active female baby weighing around $2.75 \mathrm{~kg}$ who is now 3 years old. Her past menstrual cycles were regular. She did not have any significant past medical / family history.

Her present pregnancy was a spontaneous conception 6 months after previous abortion. Dating scan gave a report of live intrauterine pregnancy of 5 weeks. Suspecting scar pregnancy, she was administered Injection Methotrexate 1 dose by a gynecologist. 2 weeks later repeat ultrasonography showed an ongoing pregnancy and she underwent MTP (Instrumental evacuation) during which she had heavy bleeding for which the procedure was abandoned. No H/o blood transfusion. Patient was discharged next day and advised to repeat ultrasound one week later. Patient presented to our causality after one week with above complaints.

$\mathrm{O} / \mathrm{E}$ in casualty patient was conscious and coherent. Patient looked severely pale with a pulse rate of 120beats per minute of low volume. BP 80/60 $\mathrm{mmHg}$ in hemorrhagic shock. Abdominal examination revealed mild distension with guarding and rigidity. LSCS scar appeared healthy. Per speculum examination revealed cervix, vagina normal except mild blood stained discharge.

Two Intravenous lines started with NS \& patient was catheterized. Blood investigation $\mathrm{Hb}-7.3 \mathrm{~g} / \mathrm{dI}$. PTINR-4.0 Rest of the blood parameters normal. USS: abdomen \& pelvis revealed a focal abnormality in lower uterine segment along anterior wall, adjacent to that a large heterogenous clot/mass of size of approximately $7.5 \times 5.9 \mathrm{~cm}$. Significant ascites was reported. Patient was posted for emergency laparotomy with consent and adequate blood in view of suspecting scar pregnancy rupture following MTP.

On laparotomy $1.5 \mathrm{~L}$ of hemoperitoneum was noted with huge clots intraperitoneally. Rent measuring 23 cmnoted anteriorly in the lower uterine segment with placental mass protruding out of uterus as a result of perforation. Bleeding from rent noted. Urinary bladder was intact. The whole picture suggested morbidly adherent placenta -Placenta increta. B/L internal iliac artery ligation done followed by total hysterectomy after getting consent in view of irregular rent, friability and bleeding from perforated area, as a life saving measure. Ovaries were retained. Intra peritoneal drain was inserted. Patient was transfused 4 units of PRBC and 5units of FFP. Post-operative period was uneventful and patient was discharged on day 10 .

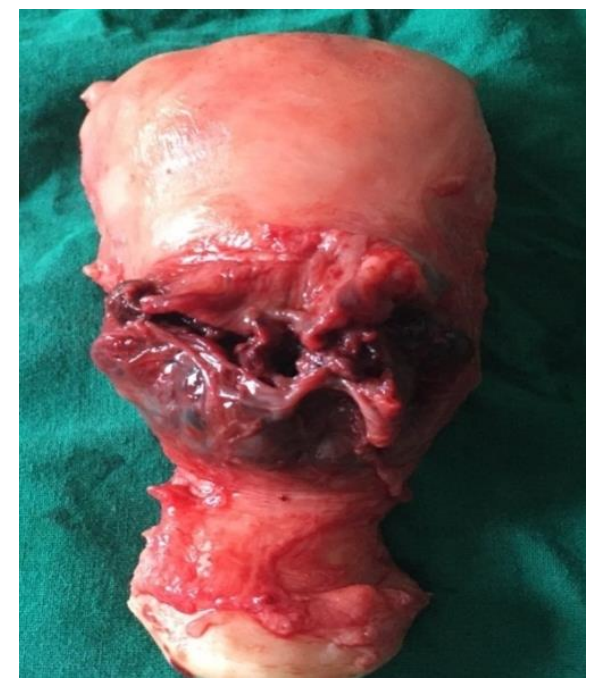

Fig. 1: Perforation of $2-3 \mathrm{~cm}$ in the LUS anteriorly noted on laporotomy 


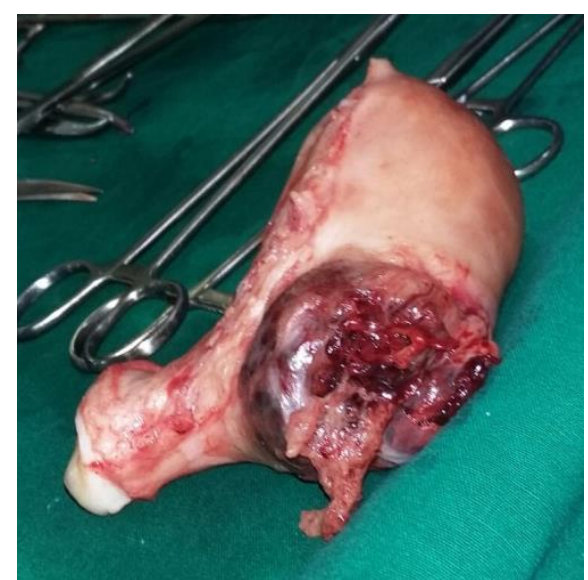

Fig. 2: Placentalmass protruding out of the uterus

\section{Discussion}

Morbidly adherent placenta should be suspected even in first trimester itself in women with known high risk factors who are undergoing MTP or suction evacuation. Early preoperative diagnosis in the suspected women is the key to save the woman's life. ${ }^{1}$ Placenta previa is an important cause of both ante partum hemorrhage and postpartum hemorrhage. ${ }^{2}$ Placenta previa has many predisposing factors. A patient of previous LSCS scar with placenta previa is of particular importance in present day obstetrics. As the number of caesarian deliveries are increasing in modern obstetrics, the number of placenta previa is also on the rise. This reinforces the significance of promoting more $\&$ more vaginal deliveries which in turn will reduce the number of LSCS and future placenta previas.

Placenta previa is associated with complications like obstetrical hemorrhage, placenta accreta and need for cesarean hysterectomy.

Morbidly adherent placenta is defined as an abnormal adherance either in whole or in part of the placenta to the underlying uterine wall. ${ }^{3}$ Morbidly adherent placenta is a general term that includes placenta accreta, increta and percreta. It is a life threatening emergency requiring a multidisciplinary approach in its management.

Adherent placenta are classified as accreta (villi are attached to myometrium), increta (villi invade myometrium), and percreta (villi invades upto serosa or into adjacent organs). Various subtypes depending on extent of placental tissue involved are total (entire placenta is adherent), partial (some placental parts adherent) and focal (a single cotyledon adherent). In unscarred uterus, occurrence of adherent placenta is rare.

Placenta accreta is the leading cause for PPH and emergency hysterectomy. Maternal mortality is as high as $7-10 \%$. Perinatal complications are also associated at times.

\section{Conclusion}

A high index of suspicion is required in the antenatal diagnosis of adherent placenta. No accurate diagnostic test is available. Final diagnosis is made intraoperatively and histologically. We need a multidisciplinary approach with good surgical expertise, plenty of blood, good interventional radiology in reducing maternal mortality and morbidity. ${ }^{3}$

A patient presenting with repeated bleeding per vaginum with a low lying placenta in the earlier weeks of gestation in previous LSCS placenta accreta has to be strongly suspected. Ultrasound along with MRI may have to be done in such cases.

In case of morbidly adherent placenta, one should resort to hysterectomy sooner rather than later. Intraoperatively bleeding is rarely a problem until an attempt is made to remove the placenta. ${ }^{4}$ Early in the antenatal period itself, from the history and ultrasound, monitoring of patients at high risk for morbidly adherent placenta has to be done.

Conflict of Interest: The authors report no conflict of interest.

Informed Consent Informed consent was obtained from the patient to prepare this manuscript. No personal information is shared in the description of this case.

\section{References}

1. Richa Aggarwal, Amita Suneja, Neelam Bala Vaid, Ponam Yadav, Abha Sharma and Kiran Mishra. Morbidly Adherent Placenta: A critical Review. J Obstet Gynaecol India.2012 Feb;62(1):57-61.

2. Arul Anne Roses, Ushadevi G. Previous lower segment ceasarean section-a potential risk factor for placenta previa. International journal of medical research and review 2015;3(4):385-389.

3. Dr Uma Satyasri Yeeda, Dr. Aruna Gollapalli \& Dr Padmavathi Thatavarthi. Placenta percreta. A case report. J Med Scix Res.2015;3(2):87-89.

4. Leena Wadhwa, Sangeetha Gupta, Prathibha Gupta, Bhawna Satiya, Rupali Khanna. Morbidly adherent placenta (MAP) lessons learnt. Open journal of obstetrics \& Gynecology, 2013;3,217-221. 